\title{
Sexualidad y autoestima en personas con dolor crónico
}

\author{
MARTÍN SÁNCHEZ GÓMEZ \\ al225771@uji.es \\ ESTEFANÍA RUIZ-PALOMINO \\ eruiz@ipsic.es \\ RAFAEL BALLESTER-ARnAL \\ rballest@uji.es
}

\section{Resumen}

Introducción: El estudio de la sexualidad y la autoestima en personas que padecen dolor crónico es de vital importancia a la hora de conocer el modo en que afrontan sus relaciones sexuales. Por otro lado, la satisfacción sexual constituye una dimensión importante de la calidad de vida, tan afectada en estos pacientes. Nuestro objetivo ha sido analizar la sexualidad de las personas con dolor crónico y su su autoestima y conocer qué factores sociodemográficos y médicos guardan relación con estas dimensiones. Método: Se administraron dos cuestionarios a una muestra de 62 personas (32 mujeres y 30 hombres) afectadas por dolor crónico y con edades comprendidas entre los 20 y 67 años (media $=46$, DT $=9,8$ ). Evaluamos los problemas sexuales y la afectación sexual mediante el cuestionario de sexualidad para pacientes de dolor crónico (Ballester y Ruiz, 2010) y la autoestima mediante el Self-esteem Scale de Rosenberg (Rosenberg, 1965). Resultados: Los resultados obtenidos en personas que padecen dolor crónico muestran afectación en la sexualidad, especialmente en la subdimensión referente a las relaciones sexuales, y en la autoestima, altamente relacionada con la dimensión problemas sexuales y la variable «Seguimiento de tratamiento psicológico». El sexo femenino, una edad avanzada y el dolor crónico derivado de la fibromialgia correlacionan negativamente con salud sexual y autoestima. Conclusión: A pesar de que existe una carencia palpable de la investigación sobre la sexualidad de las personas que padecen una dolencia crónica, es necesario incluir esta variable en los estudios sobre su calidad de vida y en los programas de intervención psicológica.

Palabras clave: dolor crónico, sexualidad, sexo y autoestima.

\section{Abstract}

Introduction: Studying sexuality and self-esteem in people with chronic pain is very important to know how they face their sexual relations. Sexual satisfaction is an important dimension of quality of life, which is greatly affected in these patients. Our objec- 
tive was to analyse the sexuality and self-esteem of people with chronic pain, and to know what socio-demographic and medical factors are related to these dimensions. Method: Two questionnaires were administered to a sample of 62 people (32 women and 30 men) affected by chronic pain, aged between 20 and 67 years (average $=46$, $\mathrm{SD}=9.8$ ). We evaluated sexual problems and sexual involvement by the Sexuality Questionnaire for Chronic Pain Patients (Ballester \& Ruiz, 2010) and self-esteem by the Self-Esteem Scale (Rosenberg, 1965). Results: The results showed that chronic pain affected sexuality, especially in the subdimension concerning sex. Self-esteem was also affected, and was highly related to sexual problems and psychological treatment monitoring. Female gender, advanced age, and chronic pain from fibromyalgia correlated negatively with sexual health and self-esteem. Conclusion: As research on the sexuality of people with a chronic health condition is evidently lacking, it is necessary to include this variable in quality of life studies and in psychological intervention programmes.

Keywords: Chronic pain, sexuality, sex, self-esteem.

\section{Introducción}

La Asociación Internacional para el Estudio del Dolor (IASP) define el dolor como una experiencia sensorial y emocional desagradable asociada a un daño actual o potencial. La misma organización, con el respaldo de la Organización Mundial de la Salud (OMS) y la Federación Europea de Secciones de la IASP (EFIC), considera el dolor crónico como la mayor amenaza para la calidad de vida a nivel mundial, amenaza que va en aumento de forma paralela al aumento de la esperanza de vida (OMS, 1996).

Desde una perspectiva biopsicosocial, se asume que el dolor es un fenómeno complejo y subjetivo en el que intervienen diversos factores biológicos, psicológicos y sociales, poco entendido y en muchas ocasiones sin resolver (IASP, 1986). Se trata de un serio problema sanitario con importantes consecuencias de sufrimiento y deterioro en calidad de vida de los pacientes, consecuencias que afectan a su entorno familiar, laboral y social; y tiene, además, serias repercusiones socioeconómicas y laborales para la sociedad (IASP, 1986). La presencia de dolor es fuente de trastornos conductuales y psicológicos importantes que precisan atención y tratamiento especializado (Wolfe, 1997). Estamos ante un problema de elevada prevalencia, insuficientemente controlado y con importantes repercusiones sanitarias y socioeconómicas (Rull, 2004). Y si todo ello es cierto, todavía lo es más cuando el dolor se prolonga a lo largo del tiempo convirtiéndose en una dolencia crónica.

Técnicamente se considera dolor crónico aquel que se prolonga durante más de seis meses y afecta la forma en que una persona lleva a cabo su vida cotidiana (Merskey, 1986). Desde el punto de vista epidemiológico, el dolor crónico es un problema extenso que afecta a 1 de cada 5 individuos; la prevalencia en España se sitúa en el $19 \%$, alcanzando los 9 millones de afectados (Casals y Samper, 2004). Según las mismas fuentes, 1 de cada 3 individuos con dolor crónico son incapaces o tienen muchas limitaciones para llevar una vida independiente. En el $25 \%$ de los casos, los pacientes consideran que el dolor crónico supone el distanciamiento o la destrucción de las relaciones con familia y amigos. El dolor crónico cambia por completo la vida de las personas que lo sufren y sigue siendo un problema de salud sin resolver. Resulta evidente que el dolor crónico tiene un elevado impacto en la vida de quien lo padece, en sus 
familias y en la sociedad. Más concretamente, la fibromialgia es un trastorno de la modulación del dolor caracterizado por un dolor crónico generalizado, donde hay una afectación muscular predominante y sensibilidad en distintos puntos del cuerpo que se asocia con fatiga, trastornos del sueño, alteraciones cognitivas y otros síntomas somáticos variables (De Gracia, 2001). Su etiología y patogénesis no es clara todavía. La fibromialgia presenta una elevada comorbilidad con otros síndromes de sensibilización central que incluye entidades como el síndrome del intestino irritable, la disfunción temporomandibular, el dolor de espalda idiopático, el síndrome de sensibilidad química múltiple, cefalea, migraña y el síndrome de fatiga crónica. Entre la fibromialgia y estos síndromes parece existir una relación bidireccional (Aaron, 2001). Esta enfermedad tiene también un impacto negativo en las relaciones personales, el desarrollo profesional y la salud mental, aunque el tratamiento psicológico atenúa los problemas en diferentes variables mentales (Bernard, 2000).

Por su parte, el concepto calidad de vida se ha relacionado con dolor crónico y cada vez está recibiendo mayor atención por parte de los clínicos e investigadores. Estudios como el realizado en Brasil (Pereira y cols., 2014) señalan que, a menor intensidad y menor número de episodios de dolor crónico, se encontraba una mejor autopercepción de la salud y una mayor satisfacción con la vida en aquellas personas que lo padecían. Se encontraron diferencias de género en los patrones de las relaciones de la depresión, la actividad y el dolor. Para las mujeres, la depresión estaba estrechamente relacionada con el informe del dolor, mientras que para los hombres la depresión fue más fuertemente relacionada con la reducción de la actividad (Haley, 1985). Gracias a este estudio, los investigadores comenzaron a considerar las diferencias de género en sus investigaciones con pacientes con dolor crónico. La baja autoestima se ha asociado con muchas condiciones de salud crónicas, además de mostrar una indirecta pero fuerte implicación negativa en el pronóstico clínico (Hegarty, 2014). Esto podría deberse a que la baja autoestima ejerce interferencia en las actividades de los enfermos y limita ciertas esferas de su vida. Kreitler (1999) propone que el dolor continuo aumenta con la edad y correlaciona con un mayor uso de servicios de salud y baja autoestima. Otra muestra de la importancia del dolor crónico en la autoestima la encontramos en el estudio llevado a cabo por Diana Elton (Elton, 1978) en que las puntuaciones de autoestima de veinte pacientes con dolor crónico se compararon con los resultados de veinte pacientes con dolor orgánico agudo y otros dos grupos de veinte personas, un grupo control de adultos y otro de estudiantes. Los resultados arrojaron que las puntuaciones de autoestima de los pacientes con dolor crónico fueron significativamente inferiores a los de los otros tres grupos, que no mostraron diferencias significativas entre sí.

Por otra parte, dentro de la calidad de vida general, la salud sexual constituye una dimensión importante (Lindau, 2010). Estudios en distintos países utilizando muestras de población general y muestras clínicas han puesto de manifiesto que la mayoría de los hombres y las mujeres consideran de gran importancia el bienestar sexual (Basson, 2007). La disfunción sexual es la manifestación más frecuente de una enfermedad física (Rico-Villademoros, 2014). Esto afecta especialmente a pacientes crónicos, con niveles de afectación todavía mayores a los estudiados en población general, afectando además a la pareja de forma muy directa. En una encuesta realizada en EE. UU. para evaluar el impacto de la fibromialgia en la salud general y la salud reproductiva, se observó que las mujeres con fibromialgia tenían significativamente menos excitación, más dolor durante el coito, menos orgasmos y menos actividad sexual que aquellas sin fibromialgia (Shaver y cols., 2006). En el estudio de Ryan y cols. (2008) el $63 \%$ de las mujeres encuestadas señalaron que la fibromialgia había alterado sus relaciones sexuales y el $71 \%$ que la enfermedad limitaba de alguna manera la realización del coito. El $80 \%$ de estas mujeres consideraban que el sexo era un aspecto importante de sus vidas. Orellana y cols. (2008) realizaron un estudio en España para evaluar la prevalencia de disfunción sexual en 
mujeres con fibromialgia en comparación con controles sanas. El $97 \%$ de las mujeres con presentaban disfunción sexual, en comparación con un $55 \%$ de las mujeres del grupo control.

En otro estudio (Izquierdo y cols., 2007) se evalúo a un grupo de mujeres sometidas a tratamiento oncológico y se demostró que las pacientes lograron recuperar una vida sexual satisfactoria, aunque con afectaciones del deseo, la autoestima y la excitación respecto a niveles anteriores a la enfermedad. En este caso, la enfermedad no ha impedido el mantenimiento de relaciones sexuales, aunque sí ha reducido la satisfacción que estas producen.

Estos estudios muestran que los pacientes con dolor crónico, en especial fibromialgia, presentan una elevada frecuencia de disfunción sexual y ciertas deficiencias en su satisfacción sexual. De todas formas, estos datos deben ser tomados con cautela y su generalización está limitada, ya que ningún estudio incluyó hombres y la mayoría fueron realizados en poblaciones muy seleccionadas.

El dolor crónico no ha recibido toda la atención que debería; por tanto, es urgente responder de forma coordinada a las necesidades y demandas de este colectivo de pacientes. $Y$ todavía han recibido menos atención algunas dimensiones fundamentales para la calidad de vida de estas personas como su vivencia de la sexualidad. Por todo ello, esta investigación pretende conocer cómo es la sexualidad de las personas con dolor crónico y si esta condición médica interfiere en la misma.

El primer objetivo de este trabajo es analizar la sexualidad de las personas con dolor crónico, evaluada a través de distintas dimensiones como relaciones sexuales, afectividad, comunicación, satisfacción y respuesta sexual.

El segundo objetivo de la investigación es analizar la autoestima de las personas afectadas por dolencias crónicas.

El último objetivo es conocer qué factores sociodemográficos y médicos guardan relación con la salud sexual y la autoestima de personas con dolor crónico.

En cuanto a lo que respecta a las hipótesis nos planteamos las siguientes:

- Hipótesis 1: las personas afectadas por dolor crónico presentarán afectación en diferentes dimensiones de su sexualidad.

- Hipótesis 2: la autoestima de personas afectadas por dolor crónico estará en niveles bajos.

- Hipótesis 3: diferentes factores sociodemográficos y médicos estarán en relación con la salud sexual y la autoestima de personas con dolor crónico.

\section{Método}

\section{Participantes}

La recogida de datos original nos dejó una muestra de 172 sujetos pero, tras eliminar del estudio todas aquellas personas que no habían realizado correctamente el cuestionario, se obtuvo una muestra final de 161 pacientes con algún tipo de dolor crónico. De estos 161, consideramos adecuado seleccionar a un número similar de hombres y mujeres, ya que de este modo podríamos comparar diferencias entre sexos controlando variables extrañas. Tradicionalmente, los estudios sobre sexualidad analizan separadamente hombres y mujeres por las grandes diferencias que se suelen dar en su vivencia de la sexualidad. Por tanto, la muestra final estuvo compuesta por 62 sujetos, todos ellos procedentes de distintas asociaciones de enfermos crónicos. Todos los participantes aceptaron colaborar voluntariamente en el estudio. 
El rango de edad estaba comprendido entre 20 y 67 años, con una media de edad de 46,1 años (DT $=9,82$ ). El estado civil de los participantes era mayoritariamente el de casado $(66,9 \%)$, el $73,8 \%$ tenía al menos un hijo y la edad media de estos era de 20,4 años (DT $=9,03$ ). El $77,7 \%$ de la muestra indicó tener estudios más allá del título de graduado escolar. El 30,7 \% de la muestra declaró estar en activo, mientras que el 69,3\% restante estaba compuesto por incapacitados $(26,2 \%)$, desempleados $(23,8 \%)$, amos de casa $(13 \%)$, jubilados $(4,6 \%)$ y estudiantes (1,6\%). En cuanto al nivel socioeconómico, el 66,9 \% declaró situarse en un nivel medio. En el ámbito de las creencias religiosas, el grupo más abundante fue el de católicos no practicantes (45,4\%). Por último, en lo relativo a las variables médicas, el $86,1 \%$ padecía fibromialgia, en término medio, desde hace 11 años ( $D T=7,88$ ) mientras que el 13,9 \% restante padecía otro tipo de enfermedades crónicas como espondilitis, enfermedades cardíacas y respiratorias, etc. desde hace una media de 11 años y medio (DT $=8,67$ ).

\section{Instrumentos}

Protocolo de datos. Para la obtención de la información descriptiva utilizamos un protocolo de datos ad hoc dividido en dos partes. En la primera (10 ítems) se preguntó sexo, edad, estado civil, número de hijos y edad de los mismos, nivel de estudios, religión, profesión, situación laboral actual y nivel socioeconómico. En la segunda parte (18 ítems) se obtuvieron los datos referentes a la enfermedad y el tratamiento de cada persona.

Cuestionario de sexualidad para pacientes de dolor crónico (Ballester y Ruiz, 2010). Utilizamos este cuestionario para evaluar la sexualidad en personas que padecen dolor crónico. Se divide en dos bloques: el primero (30 ítems, $\alpha=, 94)$ evalúa las subdimensiones de relaciones sexuales $(\alpha=, 87)$, afectividad $(\alpha=, 90)$, comunicación $(\alpha=, 92)$, satisfacción $(\alpha=, 91)$ y problemas en la respuesta sexual $(\alpha=, 89)$; el segundo (8 ítems, $\alpha=, 91$ ) evalúa la atribución que los sujetos le dan al dolor en sus problemas sexuales. El cuestionario consta de 38 ítems, 10 de los cuales son inversos. El a de Cronbach general obtenido en nuestro estudio fue ,93.

Self-esteem Scale de Rosenberg (Rosenberg, 1965). Evalúa la autoestima general con 10 afirmaciones que aluden a sentimientos globales de autovaloración (en general, estoy satisfecho conmigo mismo), 5 de los cuales están redactados en sentido positivo y 5 en sentido negativo. La fiabilidad obtenida en nuestro estudio, evaluada a través de la consistencia interna, también es buena $(\alpha=, 89)$.

\section{Procedimiento}

Tras un primer contacto con más de 30 asociaciones españolas de pacientes crónicos, pudimos contactar en segunda instancia a través de correo electrónico y teléfono con los responsables de varios centros (AVAFI, ADEC, AVAFAS, APAFIMA y AFIBROSE), donde explicamos el objetivo del estudio y pudimos llevar a cabo la administración del cuestionario. Los participantes declararon su consentimiento para participar en esta investigación y se les explicó que los datos iban a ser utilizados de manera anónima y confidencial.

\section{Análisis de datos}

Una vez obtenidas las respuestas, se procedió a almacenar los datos en la base estadística y posteriormente a su análisis mediante el programa estadístico sPSs (versión 21.0). Efec- 
tuamos un análisis de fiabilidad para conocer la consistencia interna mediante el coeficiente alfa de Cronbach; obtuvimos correlaciones bivariadas mediante la $r$ de Pearson; la diferencia de medias mediante la $t$ de Student para muestras independientes y ANOVA en el caso que hubiera más de dos variables implicadas en cada caso.

\section{Resultados}

En primer lugar, trataremos la información acerca de características médicas de los participantes. En segundo lugar, atenderemos a la afectación sexual y en autoestima. En tercer lugar, analizaremos las correlaciones obtenidas entre las variables de estudio. Por último, explicaremos los resultados obtenidos en los análisis diferenciales realizados mediante prueba $t$ de Student y ANOVA.

\section{Características médicas}

Partiendo de la base de que todos los participantes estaban afectados por un dolor crónico, el $86,2 \%$ de ellos padecía fibromialgia, mientras que el 13,8 \% restante sufría otro tipo de dolencia crónica. El tiempo medio desde que este dolor comenzó se sitúa en los 11 años (DT $=7,88$ ). Por otra parte, se les preguntó a los participantes si sus parejas sentimentales padecían algún tipo de enfermedad crónica, a lo cual contestaron afirmativamente el 10,8 \%. En cuanto al tratamiento, el $80 \%$ de la muestra declaró tomar fármacos, siendo el tipo más usado los analgésicos (52,4\%). En lo que respecta al aspecto psicológico, el 47,7\% de los participantes están inmersos en algún tipo de tratamiento; de ellos un 66,1\% utilizan psicofármacos, mientras que el 33,9 \% restante acude regularmente a terapia clínica de tipo psicológico.

\section{Afectación sexual y en autoestima}

El análisis de frecuencias y descriptivos de los ítems relacionados con la sexualidad permite apreciar datos clínicamente relevantes. Los ítems en que se han encontrado puntuaciones medias más extremas, indicando de esta forma mayor afectación, han sido el 2 («Tengo relaciones sexuales más de una vez por semana», estando el 63,8 \% nada de acuerdo con la afirmación), el 1 ("Considero que mi vida sexual es activa», con un 46,2 \% nada de acuerdo con la afirmación) y el 20 («Estoy satisfecha con mi vida sexual», estando el 45,4 \% nada de acuerdo).

En cuanto a los resultados en autoestima obtenidos en el cuestionario de Rosenberg, observamos una puntuación media de 26,02 en hombres y de 24,89 en mujeres. Comparando estas puntuaciones con los baremos en población española, observamos que tanto los hombres como las mujeres del estudio se encuentran una desviación típica por debajo de la media, $32,53(\mathrm{DT}=3,92)$ y $31,14(\mathrm{DT}=4,55)$ respectivamente.

\section{Relación entre variables sociodemográficas y médicas con problemas sexuales y autoestima}

En la tabla 1 podemos observar la correlación relativa a las variables sociodemográficas (del número 1 al 5), variables médicas (6) y las variables dependientes del estudio: problemas sexuales (de 7 a 8 ) y autoestima (9). 
Destacaremos únicamente las correlaciones observadas en las variables dependientes del estudio. En primer lugar, la puntuación total de «Problemas sexuales» correlaciona positiva y significativamente con edad $(, 21)$, número de hijos $(, 19)$ y afectación sexual atribuida al dolor $(, 61)$, además de hacerlo con todas las subdimensiones sexuales evaluadas en el cuestionario (variables $7.1,7.2,7.3,7.4$ y 7.5 ), mientras que correlaciona significativa y negativamente con nivel de estudios $(-, 18)$ y autoestima $(-, 36)$. En segundo lugar, la puntuación total en «Afectación sexual atribuida al dolor» correlaciona positiva y significativamente con tiempo desde que la pareja tiene dolor crónico $(, 55)$, con el constructo problemas sexuales $(, 61)$ y todas sus respectivas subdimensiones, además de hacerlo significativa y negativamente con autoestima $(-, 23)$. Por último, "Autoestima», además de correlacionar negativa y significativamente con problemas sexuales $(-, 36)$ también lo hace con todas las correspondientes subdimensiones y con la dimensión «Afectación sexual atribuida al dolor» $(-, 23)$.

Tabla 1

Correlación de las variables de estudio (Pearson)

\begin{tabular}{|c|c|c|c|c|c|c|c|c|c|c|c|c|c|}
\hline Variable & 1 & 2 & 3 & 4 & 5 & 6 & 7 & 7.1 & 7.2 & 7.3 & 7.4 & 7.5 & 8 \\
\hline \multicolumn{14}{|l|}{ 1. Edad } \\
\hline 2. Núm. hijos &, $44^{* *}$ & & & & & & & & & & & & \\
\hline 3. Edad hijos &, $25^{*}$ &,$- 40^{* *}$ & & & & & & & & & & & \\
\hline 4. Estudios &,- 08 &,- 15 &,- 13 & & & & & & & & & & \\
\hline 5. Socioeconómico & ,02 &,- 05 &, 09 &, $29^{* *}$ & & & & & & & & & \\
\hline 6. Enf. crónica &, $36^{* *}$ & ,06 &, 02 &,- 05 &, 12 & & & & & & & & \\
\hline 7. Problema sex. &, $21^{*}$ &, $19^{*}$ & ,11 &,$- 18^{*}$ &,- 11 &,- 06 & & & & & & & \\
\hline 7.1 Rel. sex. &, $31^{* *}$ &, $34^{* *}$ &, $14^{*}$ &, 04 &, 06 & ,08 &, $78^{* *}$ & & & & & & \\
\hline 7.2 Afectividad &, $32^{* *}$ &, $36^{* *}$ & ,11 &,$- 17^{*}$ &,$- 20^{*}$ &,- 01 &, $60^{* *}$ & $.39^{\star \star}$ & & & & & \\
\hline 7.3 Comunicación &, $20^{*}$ &, $19^{*}$ & 0,2 &,$- 22^{*}$ &,- 09 &,- 01 &, $74^{* *}$ &, $42^{* *}$ &, $53^{* *}$ & & & & \\
\hline 7.4 Satisfacción &, 11 &, $18^{*}$ & ,06 &,- 10 &,- 16 &, 05 &, $71^{* *}$ &, $34^{* *}$ &, $49^{* *}$ &, $67^{* *}$ & & & \\
\hline 7.5 Respuesta sex &, $20^{*}$ & ,10 &, $27^{*}$ &,- 11 &,- 04 &,- 03 &, $84^{* *}$ &, $27^{* *}$ &, $31^{* *}$ &, $47^{* *}$ & $.55^{* *}$ & & \\
\hline $\begin{array}{l}\text { 8. Afectación por } \\
\text { dolor }\end{array}$ &, 17 &, 10 & ,01 &,- 13 &,- 05 &,- 01 &, $61^{* *}$ &, $34^{* *}$ &, $18^{*}$ &, $30^{\star *}$ &, $34^{* *}$ &, $71^{* *}$ & \\
\hline 9. Autoestima & ,16 &,- 08 &, 15 &, 17 & , 15 & ,23 &,$- 36^{* *}$ &,$- 33^{* *}$ &,$- 29 * *$ &,$- 20^{*}$ &,$- 25^{\star *}$ &,$- 32^{* *}$ &,$- 23^{* *}$ \\
\hline
\end{tabular}

** La correlación es significativa en el nivel 0,01

* La correlación es significativa en el nivel 0,05 


\section{Análisis diferenciales en función de variables sociodemográficas}

La prueba $t$ de Student relativa al nivel socioeconómico compara las puntuaciones medias entre el grupo de participantes que declararon pertenecer al nivel bajo y medio. Los análisis únicamente han encontrado diferencias significativas entre grupos en la variable autoestima $(t=-2,04, p<0,5)$, siendo mayor en el grupo de nivel medio, con un tamaño del efecto pequeño $(d=0,38)$.

En el ANOVA correspondiente al estado civil (soltero, casado y separado) se encuentran diferencias estadísticamente significativas en las puntuaciones medias de los tres grupos en la dimensión «Problemas sexuales» $(F=2,50, p<, 05)$ y en su subdimensión "Afectividad» $(F=3,98, p<, 02)$. La prueba de Scheffé nos permite conocer con precisión entre qué grupos se establecen diferencias; en la variable «Problemas sexuales» encontramos que el grupo de solteros tiene significativamente menos problemas que los otros dos grupos; del mismo modo, en la variable "Afectividad» es el grupo de solteros el que muestra puntuaciones significativamente más bajas en cuanto a problemas en afectividad.

En el ANOVA correspondiente a religión (practicante, no practicante y ateo) se han encontrado diferencias significativas entre las medias de los grupos en la dimensión «Problemas sexuales» $(F=3,65, p<, 02)$ y la subdimensión «Relaciones sexuales» $(F=3,78, p<, 02)$. El grupo de ateos ha obtenido una puntuación significativamente inferior a la del resto de grupos en la variable «Problemas sexuales». En la variable «Relaciones sexuales», el grupo de ateos tiene significativamente menos problemas que el grupo de no practicantes, el cual, a su vez, también tiene menos problemas que el grupo de religiosos practicantes.

\section{Análisis diferenciales en función de variables médicas}

La prueba $t$ de Student relativa a la presencia o no de dolor crónico en la pareja sentimental revela diferencias en las puntuaciones medias entre el grupo de participantes en que su pareja padece una afectación crónica frente a los participantes que no. Existen diferencias significativas entre grupos en la dimensión «Problemas sexuales» $(t=1,18, p<, 04)$ y la subdimensión «Afectividad» $(t=1,77, p<, 05)$ En ambos casos, el tamaño del efecto es moderado $(d=0,57)$ y es el grupo sin dolor crónico en la pareja el que presenta puntuaciones menos afectadas.

En la prueba $t$, con respecto al seguimiento o no de tratamiento, encontramos diferencias significativas entre ambos grupos en lo que respecta a las subdimensión «Relaciones sexuales» $(t=1,85, p<, 04)$ con un tamaño del efecto moderado $(d=, 58)$ y la dimensión "Afectación sexual debida al dolor» $(t=1,79, p<, 05)$ y un tamaño del efecto también moderado $(d=0,49)$. El grupo que no sigue tratamiento muestra puntuaciones, en promedio, más bajas en ambas variables. Esto indica la presencia de menos problemas en las relaciones además de una menor afectación derivada del dolor.

Con respecto al seguimiento o no de tratamiento para sus problemas psicológicos, los resultados obtenidos evidencian diferencias que alcanzan cotas significativas en la dimensión «Autoestima» $(\mathrm{t}=-2,76, \mathrm{p}<, 001)$ con un tamaño del efecto moderado $(\mathrm{d}=, 49)$. El grupo que sigue tratamiento muestra puntuaciones más altas, lo cual viene a indicar mayor nivel de autoestima. 


\section{Discusión y conclusiones}

El primer objetivo de este trabajo era analizar la sexualidad de laspersonas con dolor crónico, evaluada a través de distintas dimensiones como relaciones sexuales, afectividad, comunicación, satisfacción y respuesta sexual. Los resultados obtenidos muestran que la esfera sexual de los participantes se halla afectada de forma importante, especialmente la subdimensión referente a las relaciones sexuales. Los problemas sexuales encontrados están en relación con la edad, el número de hijos, el nivel de estudios, el estado civil, la religión, la existencia de dolor crónico en la pareja, el tipo de tratamiento farmacológico, el uso de tratamiento alternativo, la coexistencia de enfermedades crónicas y la autoestima.

El segundo objetivo de la investigación era analizar la autoestima de las personas afectadas por dolencias crónicas. Los resultados señalan que la autoestima de estas personas se encuentra en niveles por debajo de lo que cabría esperar. Esta problemática está relacionada con el nivel socioeconómico, la adherencia al tratamiento psicológico, la afectación sexual derivada del dolor y los problemas sexuales.

El tercer y último objetivo era conocer qué factores sociodemográficos y médicos guardan relación con la salud sexual y la autoestima. Como más arriba hemos detallado, se ha demostrado que tanto factores sociodemográficos (edad, nivel de estudios, religión) como médicos (seguimiento de un tratamiento farmacológico, psicológico y alternativo; tiempo desde que comenzó su dolencia y la de su pareja; existencia y modalidad de un dolor crónico secundario, y la coexistencia de otra enfermedad no crónica) influyen directamente en las relaciones sexuales y la autoestima.

Tras haber analizado los resultados de forma pormenorizada, podemos decir que se encuentran en consonancia con las hipótesis planteadas al principio del trabajo. Las personas afectadas por dolor crónico presentan afectación en diferentes dimensiones de su sexualidad; la autoestima de estas personas se encuentra en niveles bajos; diferentes factores sociodemográficos se relacionan con la salud sexual y la autoestima y, por último, diferentes factores médicos están en relación con la salud sexual y la autoestima de personas con dolor crónico.

Uno de los problemas encontrados durante el estudio fue el tamaño muestral. Las dificultades para encontrar participantes puede deberse a la falta de incentivos por participar en el estudio o bien por la complejidad propia de encontrar una muestra clínica. Los estudios futuros deben tratar de continuar con la línea aquí propuesta e intentar encontrar la igualdad de género con el fin de obtener resultados más estables, de esta manera será posible generalizar los datos a toda la población afectada y no solo a las mujeres como se ha estado realizando mayoritariamente hasta la fecha.

\section{Referencias bibliográficas}

Aaron, L. A. y Buchwald, D. (2001). A review of the evidence for overlap among unexplained clinical conditions. Annals of Internal medicine, 134, 868-881.

Ballester, R. y Ruiz, E. (2010). Cuestionario de sexualidad para pacientes de dolor crónico. Manuscrito no publicado.

Basson, R. (2007). Sexuality in chronic illness: no longer ignored. The Lancet, 369, 350-352.

Bernard, A. L., Prince, A. y Edsall, P. (2000). Quality of life issues for fibromyalgia patients. Arthritis Care \& Research, 13, 42-50.

Bonet, J. V. (1997). Sé amigo de ti mismo: manual de autoestima. Santander: Sal Terrae.

Casals, M. y Samper, D. (2004). Epidemiología, prevalencia y calidad de vida del dolor crónico no oncológico: Estudio ITACA. Revista de la Sociedad Española del Dolor, 11, 260-269. 
De Gracia, M., Marcó, M., Ruiz, J. y Garabieta, F. (2001). Evaluación de los aspectos psicológicos de la fibromialgia. Análisis y modificación de conducta, 27, 959-980.

Elton, D., Stanley, G. V. y Burrows, G. D. (1978). Self-esteem and chronic pain. Journal of psychosomatic research, 22, 25-30.

Haley, W. E., Turner, J. A. y Romano, J. M. (1985). Depression in chronic pain patients: relation to pain, activity, and sex differences. Pain, 23, 337-343.

Hegarty, D. y Wall, M. (2014). Prevalence of stigmatization and poor self-esteem in chronic pain patients. Journal of Pain \& Relief, 3, 3-6.

International Association for the Study of Pain (1986). Classification of chronic pain. Pain, 3 , 19-32.

Izquierdo, M., Torres, B. T., González, M. y Jauregui, U. (2007). Sexualidad en un grupo de mujeres cubanas con cáncer de mama. Revista Sexología y Sociedad, 13, 19-27.

Kreitler, S. (1999). Denial in Cancer Patients: Psychosocial Issues. Cancer investigation, 17, 514-534.

Lindau, S. T. y Gavrilova, N. (2010). Sex, health, and years of sexually active life gained due to good health: evidence from two US population based cross sectional surveys of ageing. BMJ, 340, c810. doi: http://dx.doi.org/10.1136/bmj.c810

Merskey, H. E. (1986). Classification of chronic pain: Descriptions of chronic pain syndromes and definitions of pain terms. Pain, 3, 226-238.

Organización Mundial de la Salud (1996). Cancer pain relief: with a guide to opioid availability.

Orellana, C., Casado, E., Masip, M., Galisteo, C., Gratacós, J. y Larrosa, M. (2008). Sexual dysfunction in fibromyalgia patients. Clinical \& Experimental Rheumatology, 26, 663-666.

Pereira, L. V., Vasconcelos, P. P., Souza, L. A. F., Araújo, G., Nakatani, A. y Bachion, M. (2014). Prevalencia, intensidad de dolor crónico y autopercepción de la salud entre ancianos: estudio de base poblacional. Revista Latino-América Enfermagem, 22, 662-669.

Rico-Villademoros, F. (2014). Evaluación de la función sexual en mujeres y hombres con fibromialgia. Tesis doctoral. Universidad de Granada.

Rosenberg, M. (1965). Society and the adolescent self-image. Princeton, NJ: Princeton University Press.

Rull, M. (2004). Abordaje multidisciplinar del dolor de espalda. Revista de la Sociedad Española del Dolor, 11, 119-121.

Ryan, S., Hill, J., Thwaites, C. y Dawes, P. (2008). Assessing the effect of fibromyalgia on patients. Nursing Standard, 23, 35-41.

Shaver, J. L., Wilbur, J., Robinson, F. P., Wang, E. y Buntin, M. S. (2006). Women's health issues with fibromyalgia syndrome. Journal of Women's Health, 15, 1035-1045.

Wolfe, F. (1997). The relation between tender points and fibromyalgia symptom variables: evidence that fibromyalgia is not a discrete disorder in the clinic. Annals of the Rheumatic Diseases, 56, 268-271. 